Volume 8, No. 7, July - August 2017

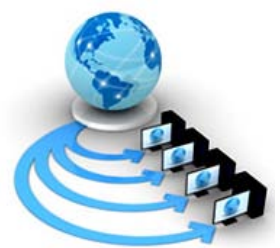

International Journal of Advanced Research in Computer Science

RESEARCH PAPER

\author{
Available Online at www.ijarcs.info
}

\title{
COMPARATIVE ANALYSIS OF BP-NN, SVM, LVQ AND RBF FOR DIAGNOSIS OF AUTISM SPECTRUM DISORDER(ASD) BASED ON DSM-IV CRITERIA
}

\author{
Lakhwinder Kaur \\ Department of Computer Science and Engineering \\ CT Group of Institutions
}

Harjit Pal Singh

Department of Electronics and Communication Engineering

CT Group of Institutions

\author{
Vikas Khullar \\ Department of Computer Science and Engineering \\ CT Group of Institutions \\ Manju Bala \\ Department of Computer Science and Engineering \\ Khalsa College Of Engineering and Technology,India
}

\begin{abstract}
Training artificial networks is a complex task of especially importance in the supervised learning field of research. Autism spectrum disorder is neurodevelopment disorder having qualitative impairments in social interaction, communication and repetitive /restrictive patterns of behavior. DSM-IV criteria is used for diagnosing autism spectrum disorder. DSM-IV (Diagnostic and Statistical Manual of Mental Disorders, 4th Edition) describes all mental health disorders for both children and adults which has been published by American Psychiatric Association .We intend to show the superiority (accuracy and error rate) of the Back-propagation Neural Network over other more — standard algorithms in neural network training. In this work we tackle the problem with four algorithms, Back-propagation Neural Network (BPNN), LVQ (learning vector quantization), SVM (support vector machine) and RBF(radial basis Function) those are implemented using MATLAB tool and try to over a set of results that could hopefully faster future comparisons by using a standard dataset of ASD children and further presents the results. Our conclusions clearly establish the advantages of the BPNN algorithm over the other algorithms in diagnosing the ASD children.
\end{abstract}

Keywords-Autism spectrum disorder(ASD), Diagnosis,DSM-IV,SVM , RBF, LVQ(learning vector quantization) and Back Propagation Neural Network (BP-NN)

\section{INTRODUCTION}

Autism Spectrum Disorder is neurodevelopment disorder having impairment in social interaction, communication and having restrictive repetitive pattern of behaviors was first described by Kanner in 1943[3], and Asperger in 1944[4].Autism Spectrum Disorder covers large span of symptoms, skills and level of impairment. Autistic children can be viewed and considered as non communicative, non interactive and non responsive. Children suffering from autism having communication problem, repeated body and motor movements, harming to themself or harming to others , giving the unusual response to people also[1,2].Some professionals use Pervasive Development Disorder (PDD) to describe Autism Spectrum Disorder. In addition to autism spectrum disorder, there are four other disorders that can also to be considered as PDD[5].

A. Asperger's syndrome: Children with this disorder also having problems with social, communication and emotional skills, as well as unusual behaviors and interests but their intelligence level is high and they take initiative to interact with other ones but not know the way accurately, they can talk alot and also make eye contact[2,5] .

B. Childhood disintegrative disorder (CDD): $\mathrm{CDD}$ is Heller's syndrome in which children grows normally up to 2 years and then suffer with a severe loss of social, behavioral and communication skills[2,5].

C. Pervasive Developmental Disorder-Not Otherwise specified (PDD-NOS): is mental disorder and also called Atypical Autism e.g. dementia due to head trauma[2,5].

D. Rett syndrome: is genetic disorder mostly occurs in girls[2].

\section{BACKGROUND}

Since last researches had proposed many medical diagnosis models based on Neural Networks.

C.S Kanimozhiselvi \& A. Pratap, 2016 explains Childhood autism a Neuro-psychiatric developmental disorder that impairs mainly three functional areas in a child: social, communication and behavior. Author demonstrates the application of a Possibilistic-Linear Vector Quantization 'Po-LVQ' neural network for the preliminary screening and grading(Normal, Mild-Moderate, Moderate-Severe, Severe) of childhood autistic disorder. The Po-LVQ result has been compared with the performance of a general LVQ and other existing applied models. Po-LVQ based childhood autism grading has been seemed to be better and can be applied for grading childhood autism while developing an autism assessment support system. It has been able to perform with an improved overall accuracy of 95\% [25].

(Marc Fakhoury, 2015) defines autism as neurodevelopment disorder having impairment in social interaction, social 
communication and restrictive repetitive behaviors. Author briefs with the various core and secondary symptoms of autistic child. The core symptoms had reduced language skills and social interaction, as well as the presence of repetitive and stereotypic behaviors and secondary symptoms include complications such as self-injury, hyperactivity, aggression, and co-occurring psychiatric disorders such as anxiety and major depression. Author also discuss the causes of ASD as various environment factor like profound changes in brain development, neurological processes, genetic factor like cognitive changes that lead to impairment in social interaction, and language development and gene environment interaction factor[22].

Nurul Ridwah et.al.(2014)had investigated autism severity level detection by using the fuzzy expert system. For the research, data has been collected from various interview sessions with clinical psychologist, distributive questions to teachers and parent with autistic children. The processes involve Fuzzy system architecture, fuzzification, rules evaluation and defuzzification. Symptoms has been disclosed using the chats e.g. CARS(childhood autism rating scale) and ADI(autism diagnostic observation) etc. Author evaluates the results using forward chaining, system testing and system validation .After results, it has been concluded that system has been able to detect autism severity level with 92\% accuracy[23].

Marisela Huerta et al. (2012) had proposed DSM-IV criteria for autism spectrum disorders(ASDs) in efforts to increase diagnostic sensitivity and specificity. This study had determined the proposed DSM-5 criteria for the single diagnostic category of autism spectrum disorder in children with DSM-IV pervasive developmental disorders (PDDs) and non-PDD. Three data sets contains 4,453 children with DSM-IV clinical PDD diagnoses and 690 with non-PDD clinical diagnoses .Data items collected from a parent report measure ASD symptoms were matched to DSM-5 criteria and used to evaluate the sensitivity and specificity of the proposed DSM-5 criteria and current DSM-IV criteria. The proposed DSM-5 criteria confirmed 91\%children with DSM-IV PDD diagnoses. Sensitivity remained high in specific subgroups, as well as in girls and children under 4 . The DSM-5 ASD specificity was determined overall 0.53 , while the specificity of DSM-IV ranged from 0.24 , for clinically diagnosed PDD-NOS to 0.53 for autistic disorder [24].

Imianvan and A.A, 2013 explained Autism as a brain disorder that is associated with a wide range of developmental problems. Author presented hybrid neurofuzzy expert system to help in diagnosing autism using a set of symptoms. Author discussed some of the symptoms as avoiding eye contact, play alone, not smiling, not responding to names, echolia, unusual language, not talking, repetitive movement, self-mutilation and reduced sensitivity to pain. The system uses fuzzified set of data and incorporated into neural network and informs the patient as an autistic sufferer. This designed system should be introduced in health care delivery centers and hospitals to ease the work of physicians[2].

Koushal Kumar and Abhishek,2012 had used Multilayer Perceptrons (BP-NN), Radial Basis Function Networks, Learning Vector Quantization algorithms for the diagnosing kidney stones and compares the results for superior one. At the end back propagation algorithm has been resulted as more accurate[7].

Shukla A and Tiwari R, 2009 had presented the comparison of three algorithms back propagation, RBF and LVQ for Diagnosing Thyroid disease and finds the best accurate for diagnosis. According to this research LVQ has been presented as $98 \%$ accuracy [8].

\section{ARTIFICIAL NEURAL NETWORKS}

Artificial Neural Network is a system represented as a set of nodes called neurons, having connection between these neurons and ANN is designed to work like the biological human brain that reproduce multiple layers of simple processing elements. Each neuron is linked to its neighbors with irregular weights that weights demonstrates strength of connection between the neurons as shown in Fig.1.1. Each neuron has an associated activation function which is performed on the input to get the output as a result. Mostly neural network architecture has three layers in its structure. First layer is input layer which gives an interface with the environment, second layer is hidden layer where computation is performed and last one is output layer where output is stored. Data is propagated through successive layers, with the final result available at the output labter.er Many different types of neural networks are available. Multi-layer perceptron is popular due to having more than one hidden layer in its structure which helps sometimes in resolving complex problems where a single hidden layer neural network cannot solve that type of complex problems [7,16]. Artificial Neural Networks are called machine learning algorithms. Output from a neuron with input $\mathrm{X}$ and weight $\mathrm{W}$ is obtained as follows in Eq. (1):

$$
\mathrm{a}_{\mathrm{k}}=\Sigma_{\mathrm{i}=1, \mathrm{n}} \mathbf{W}_{\mathrm{i}, \mathrm{k}} \mathrm{Xi} \ldots(\mathbf{1})
$$

Nowdays, mostly various Artificial Neural Networks with changeable algorithms and architectures have been successfully used for diagnosing diseases such as kidney stone diseases[7], breast cancer[17] ,heart diseases [18], diabetes [19], thyroid[8], hepetite diseases[9] etc. Proficiency of Artificial neural networks is becoming more and more popular in medical diagnosis and many more others areas. Fig. 1.1 represents artificial neural network model. [16]

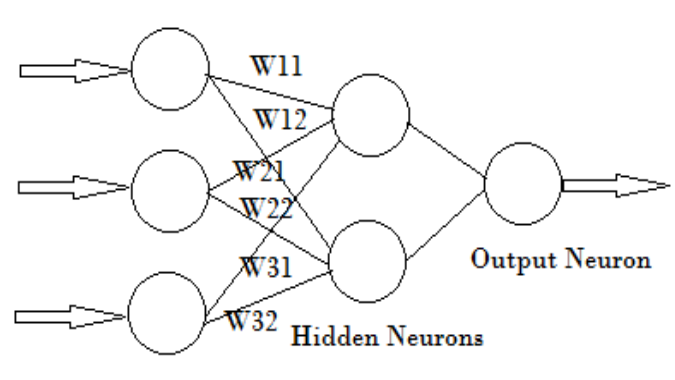

Input Neurons

Fig. 1.1 Artificial Neural Network

\section{OBJECTIVES}

i. Use The DSM-IV Criteria For Diagnosing ASD. 
ii. To Implement SVM, RBF, Back Propagation Neural Network and LVQ Algorithms and compare the parameters viz. accuracy, error rate, execution time.

\section{DIAGNOSING CRITERIA}

Various DSM's (Diagnostic and Statistical Manual of Mental Disorders)are used for diagnosing autistic disorder. DSM-I,DSM-II,DSM-III,DSM-IV,DSM-V are used for diagnosis of autism.

According to various DSM's:

i. DSM-I gives description of diagnostic criteria .Term REACTION is used in DSM-I. DSM-I views that mental disorders represents reactions of the personality to psychological, social and biological factors. DSM-I describes the acute brain syndrome and chronic symptoms[10].

ii. DSM-II gives classification on the mental disorders uses ICD-8(international classification of diseases, 8th edition). The term REACTION is not used in the DSM-II criteria. Used the DIAGNOSTIC terms for understanding of the non-organic mental disorder[11].

iii. DSM-III clinical useful for making treatment and management decisions in varied clinical settings. It maintains compatibility with ICD-9 and uses various AXES. It diagnosis the organic brain syndromes: intoxication withdrawal, delirium, dementia, amnestic syndrome, affective syndrome and personality syndrome and also considers the 'infantile autism'[12].

iv. DSM-III-R identifies the signs and symptoms such as disorientation, mood disturbance, development disorders. In DSM-III-R , ADHD is grouped with conduct disorder and oppositional deficient disorder under the rubric of disruptive behavior disorders. In this PDD grouped together with mental retardation and specific development disorder under the new rubric of development disorder[13].

v. DSM-IV describes the PDD in which autistic disorder, Rett's disorder, childhood disintegrative disorder, Asperger's disorder and PDDNOS(Pervasive Developmental Disorder-Not otherwise specified)[14].

vi. In DSM-V autism spectrum disorder(ASD) is new which encompasses the previous DSM-IV, and PDD comprises ASD, Retts disorder and childhood disintegrative disorder where ASD further comprises autistic disorder, asperger's disorder and PDD-NOS[15].

At present, diagnostic criteria according to the Diagnostic and Statistical Manual of Mental Disorders Fourth Edition (DSM-IV; American Psychiatric Association, 1994) are grouped into three main categories: impairments in social interaction, impairments in verbal and nonverbal communication, and restricted repetitive behavior of activities and interests.

In this work DSM-IV criteria is used for diagnosing autistic children. According to DSM-IV criteria rules are:

A total of six (or more) items from (1), (2), and (3), with at least two from (1), and one each from (2) and (3):
(1) Qualitative deterioration in social interaction as demonstrated by considering at least two from the following:

(a)Lack of numerous nonverbal behaviors such as eye contact, facial expression, body postures, and gestures to regulate social interaction

(b) Unable to develop peer relationships

(c)A lack of share enjoyment, interests, or achievements with other people

(d) Lack of social or emotional reciprocity

(2) Qualitative impairments in communication as manifested by at least one of the following:

(a) Lack of the development of spoken language

(b) Impairment in the ability to initiate or sustain a conversation with others

(c) Stereotyped and repetitive use of language

(d) Lack of varied, spontaneous make-believe play

(3) Repetitive and prohibitive stereotyped patterns of behavior, interests, and activities are evidenced by at least one of the following:

a. Hyperactivity or sensory movements

b. Specific nonfunctional routines

c. Stereotyped and repetitive motor mannerisms

d. Persistent preoccupation with parts of objects.

\section{DATA COLLECTION}

Data of various ASD patients are collected and from collected data ASD patients are diagnosed by using DSMIV criteria .During data collections i visualized the various autistic children and analyzed autistic children speak differently like some speak single word, some speak after taking a pause between the words.

\section{TABLE 1.1: ATTRIBUTES AND PARAMETERS USED} FOR DIAGNOSING

\begin{tabular}{|l|l|}
\hline 1 & Lack of eye contact \\
\hline 2 & Lack of social interaction \\
\hline 3 & Lack of body gestures \\
\hline 4 & Lack of facial expression \\
\hline 5 & Difficulty in Developing relationships \\
\hline 6 & Share enjoyment \\
\hline 7 & Lack of social reciprocity \\
\hline 8 & Lack of intiation of conversation \\
\hline 9 & Repetitive movements \\
\hline 10 & Different Voice rate \\
\hline 11 & Lack of pointing or play with objects \\
\hline 12 & Stereotyped and repetitive motor movements \\
\hline 13 & Some routines \\
\hline 14 & Hyperactivity \\
\hline 15 & Abnormal functioning \\
\hline
\end{tabular}

Autistic children performs repetitive movements continuously like if they start smiling then they continuously smile for a long time, continuously doing a rotation of hands, flapping hands, If they start crying , jumping then they continuously perform that activity. Some are too much fat and having thick chin. Waving and picking Hands in different ways, some are not able to walk by own self they need help or guidance by someone. They don't have too 
much capability to remember all the things when they learnt 2 or more tasks then they forget other tasks Some produced too much high sounds, some are in their own world etc.

\section{METHODOLOGY}

In this work data of various ASD patients are collected and analyzed the patients by using the DSM-IV criteria. After this data is trained and tested by SVM,BP-NN,RBF and LVQ. Find the most accurate model for the diagnosis of ASD. All the networks are simulated by using MATLAB.

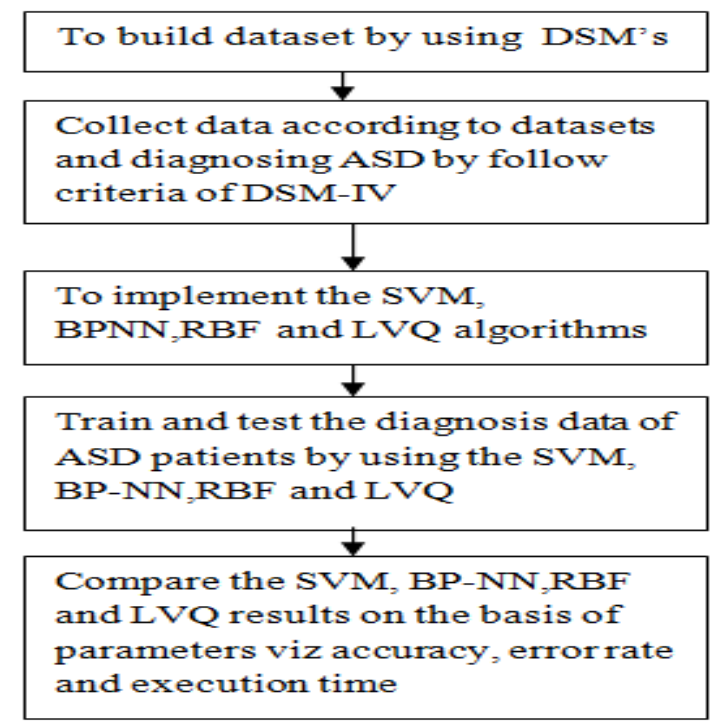

Fig. 1.2:Overall Block Diagram of the methodology used in this work

\section{VARIOUS ANN ALGORITHMS}

The main aim of this work is to compare the classification performance of artificial neural networks algorithms for the diagnoses of autism spectrum disorder disease. The algorithms used are SVM, Learning Vector Quantization (LVQ), RBF and a back propagation algorithm. A brief description of them is as follow.

\section{A. BACK PROPAGATION NEURAL NETWORK (BP-NN)}

Back propagation algorithm is also termed as multilayered feed forward network. Backpropagation neural network is a supervised learning network and it means to 'back propagate the errors'. It takes a dataset of the desired output for many inputs, for creating the training set. There are various transfer functions like sigmoid, tan sigmoid and log sigmoid[20].

The Back-propagation is a form of supervised learning for multi-layer nets, also known as the generalized delta rule. Data items having error at theOutput layer is "back propagated" to earlier ones, allowing incoming weights to these layers to be updated. It is most often used as training algorithm in neural network applications. The back propagation algorithm was developed by Paul werbos in 1974 and rediscovered by Rumelhart and parker. The back propagation algorithm has been mostly used as a learning algorithm. The common type of artificial neural network consists of three groups, or layers: input layer, hidden layer, output layer, a layer of "input" units is connected to a layer of "hidden" units, which is connected to a layer of"Output" unit. Fig 1.3 represents the BP-NN model.[27]

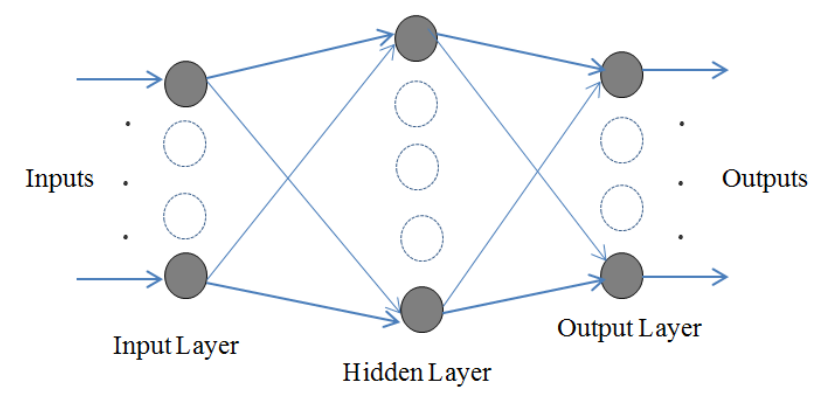

Fig.1.3: Back propagation neural network

\section{B. LEARNING VECTOR QUANTIZATION(LVQ)}

A Learning Vector Quantization Network (LVQ network) has two layers first one is competitive layer which learns to classify input vectors in much the same way as the competitive layers of Self-Organizing Nets and its considered as feature selection layer and a second layer is linear layer which transforms the competitive layer's classesinto target classifications defined by the user and this layer is considered as classification layer. The classes learned by the first layer are referred to as subclasses and the classes of the second layer as target classes. Both the competitive and linear layers have one neuron per sub or target class. LVQ learning in the competitive layer is based on a set of input/target pairs. The second layer needs no learning as the output classes (target classes) are known for each input pattern. Fig 1.4 represents LVQ model. [25]

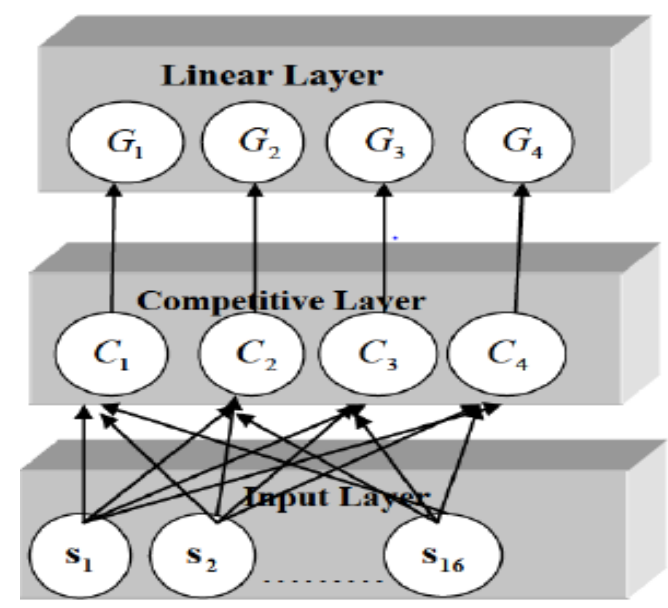

Fig.1.4: LVQ

\section{SUPPORT VECTOR MACHINE(SVM)}

Support vector machines (SVM) are basically linear classifiers or Linear Learning Machines (LLM) used for classification and regression. In SVM, ahyperplaneasseparator between two classes is 
chosen to minimize the structural risk of misclassifying by maximizing the functional gap between two classes, the training data on the marginal sides of this optimal hyper plane called support vectors. The learning process is the determination of those support vectors. For non-linearly-separable data, SVM maps the input vector from input space to some normally higher dimensional feature space by introducing Kernel functions. Precisely selection of the Kernel function is an important step is successful design of a SVM in specific classification task $[8,9]$.

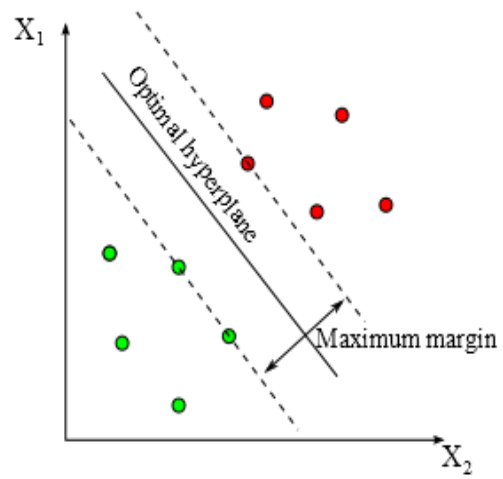

Fig.1.5: Support vector machine[26]

\section{RADIAL BASIS FUNCTION(RBF)}

Radial basis networks are powerful tools forclassification. RBF has a two-layer structure. Neurons in the first layer compute the distance of the input vector to their centers, so the first layer determines which neuron is more similar (closer) to input vector. The second layer has linear activation function and its output is weighted sum of output of first layer. Learning of RBF is much easier and faster than BP networks as network parameters in first layer (centers) are set randomly and weights of the second layer are calculated to minimize the classification error.[9]

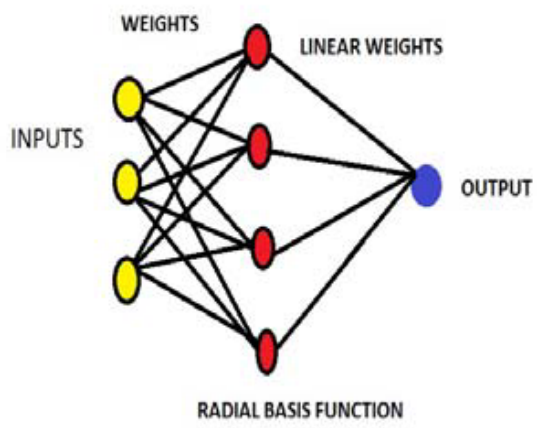

Fig.1.6: Radial basis function [9]

\section{RESULTS AND DISCUSSION}

By using the Matlab tool training and testing operations of SVM, BP-NN,RBF and LVQ are performed for diagnosing ASD. In this work I used SVM, BPNN,RBF and LVQ algorithms.I compared all the algorithms by using parameters viz accuracy, error rate(MSE), RMSE and execution time. In this back propagation gives high accuracy and less error rate as compared with SVM, LVQ and RBF. Below discussed are the parameters used for analyses.

Accuracy: Accuracy is defined as how many results are close to actual value.

Accuracy \%age=(correct results/all results)*100

Execution time: System uses how much time to run the whole program.

Error Rate: The Error Rate means to compares input data from a transmitter with input data from a receiver.

\%Error=(theoretical value-experimental value) /theoretical value

$$
M S E=1 / n \sum_{i=1}^{n}\left(y-y_{i}\right)^{2}
$$

RMSE:(Root Mean Square Error) is used for numerical predictions.RMSE is simply the square root of MSE(mean square error).

$$
\begin{aligned}
& R M S E=\sqrt{1} / n \sum_{i=1}^{n}\left(y-y_{i}\right)^{2} \\
& \mathrm{Or} \\
& R M S E=\sqrt{ } M S E
\end{aligned}
$$


TABLE 1.2: COMPARATIVE RESULTS

\begin{tabular}{|l|l|l|l|l|}
\hline ALGORITHM & $\begin{array}{l}\text { ACCURAC } \\
\text { Y }\end{array}$ & $\begin{array}{l}\text { ERROR } \\
\text { RATE(MSE) }\end{array}$ & RMSE & EXECUTION TIME \\
\hline BP-NN & 98.37 & 1.62 & 1.27 & 15.72 \\
\hline SVM & 89.47 & 10.52 & 3.24 & 0.22 \\
\hline LVQ & 71.79 & 28.20 & 5.31 & 12.55 \\
\hline RBF & 84.21 & 15.78 & 3.97 & 0.04 \\
\hline
\end{tabular}

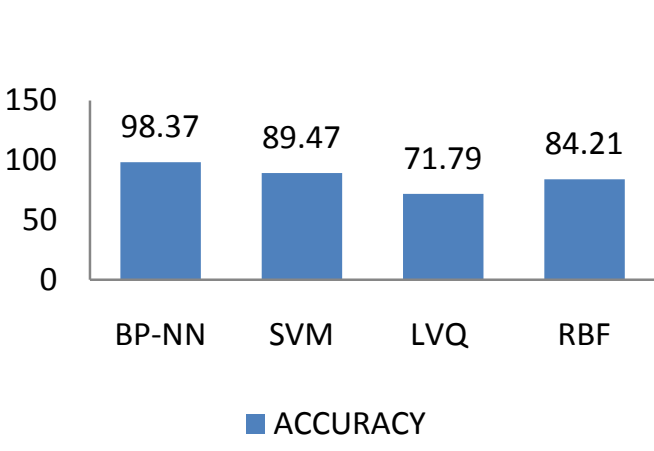

Fig.1.7: Accuracy of all algorithms

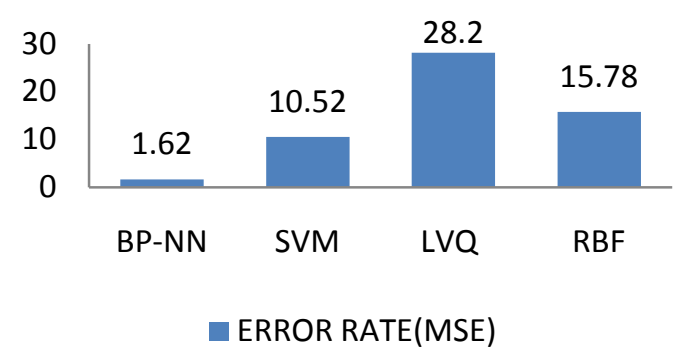

Fig.1.8: MSE of all algorithms

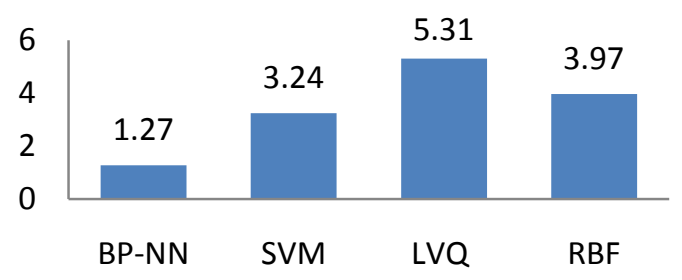

RMSE(Root Mean Square Error)

Fig.1.9: RMSE of all algorithms

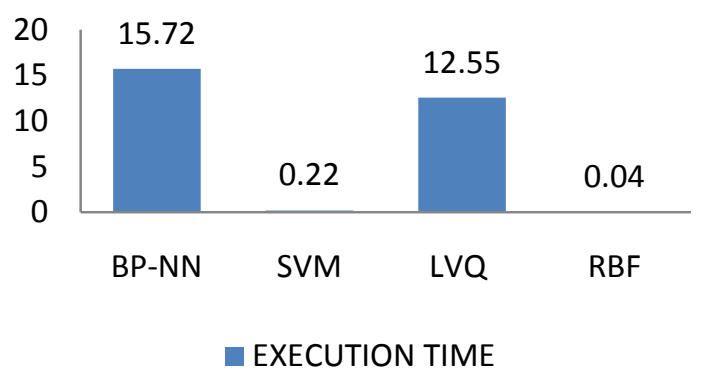

Fig.1.10: Execution time of all algorithms

\section{CONCLUSION}

A comparison of algorithms for training feed forward neural networks was done. Tests were done on four different algorithms BPNN, LVQ,SVM and RBF. Experimental results show that Back-propagation (BP-NN) outperforms all other algorithms in diagnosing autistic children with accuracy 98.37\%. Also BP-NN has less error rate than other algorithms. In future ASD disorder can be further diagnosed by using PSO and other Artificial intelligence algorithms.

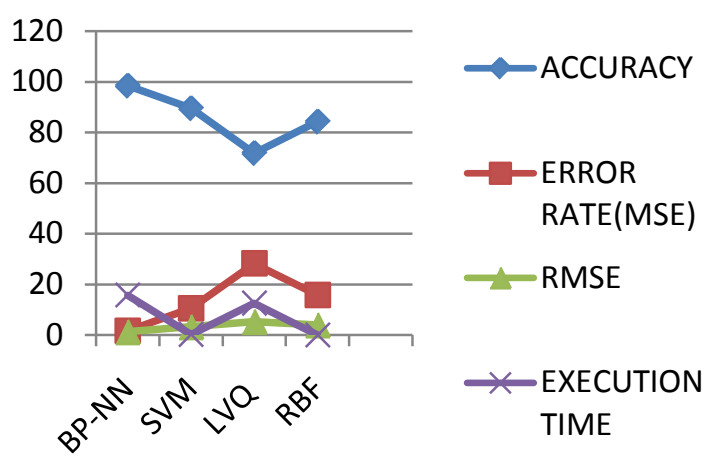

Fig.1.11: comparative results of all algorithms

\section{REFRENCES}

1. RachnaAhuja, DarvinderKaur, Neuro-Fuzzy Methodology for Diagnosis of Autism, (IJCSIT) International Journal of Computer Science and Information Technologies, Vol. 5 ,issue 2, 21712172,2014 
2. Imianvan, A.A ,intelligent neuro fuzzy expert system for autism recognition,Nigerianjournel of science and environment,vol.12,2013

3. Kanner, L. Autistic disturbances of affective contact. Nervous Child, 2, 217-250,1943.

4. Asperger, H. Die "autistischenPsychopathen" imKindesalter. Arch. PsychiatrieNervenkrankheiten, 117, 76-136. (Translated in Frith U. (Ed.) (1991). Autism and Asperger Syndrome.Cambridge: Cambridge University Press.)

5. Wrong Diagnosis, Autism, retrieved from http"//www.Wrongdiagnosis.com/l/autism/Introduction/, 2011

6. Abhishek, GourSundarMitra Thakur, Dolly Gupta, Proposing Efficient Neural Network Training Model for Kidney Stone Diagnosis, (IJCSIT) International Journal of Computer Science and Information Technologies, Vol. 3 ,issue 3, 3900-3904,2012

7. Koushal Kumar, Abhishek, Artificial Neural Networks for Diagnosis of Kidney Stones Disease, I .J. Information Technology and Computer Science, 7, 20-25,2012

8. Shukla A and Tiwari R "Diagnosis of Thyroid Disorders using Artificial Neural Networks” Department of Information Communication and Technology,2009

9. Rouhani $\mathrm{M}$ and Haghighi $\mathrm{M}$ "The Diagnosis of Hepatitis diseases by Support Vector Machines and Artificial Neural Networks” Islamic Azad University, Gonabad branch Gonabad, Iran ,2009

10. N. W. Washington,Diagnostic and statistical manual of mental disorders, DSM I(First edition),American psychiatric association, 1952.

11. N. W. Washington,Diagnostic and statistical manual of mental disorders, DSM II(second edition),American psychiatric association, 1968.

12. N. W. Washington, Diagnostic and statistical manual of mental disorders, DSM III(Third edition),American psychiatric association, 1980.

13. N. W. Washington,Diagnostic and statistical manual of mental disorders, DSM III -R(Third edition Revised),American psychiatric association, 1987.

14. N. W. Washington,Diagnostic and statistical manual of mental disorders, DSM IV(Fourth edition),American psychiatric association, 2005.

15. N. W. Washington,Diagnostic and statistical manual of mental disorders, DSM 5(fifth edition),American psychiatric association, 2013.

16. ArpneekKaur, AbhishekBhardwaj, Artificial Intelligence in Hypertension Diagnosis: A Review, (IJCSIT) International Journal of Computer Science and
Information Technologies, Vol. 5 ,issue 2, 2633-2635, 2014

17. Ahmad, Fadzil, et al. "Intelligent Breast Cancer Diagnosis Using Hybrid GA-ANN." Computational Intelligence, Communication Systems and Networks, Fifth International Conference on. IEEE,2013.

18. Amin, Syed Umar, KavitaAgarwal, and Rizwan Beg. "Genetic neural network based data mining in prediction of heart disease using risk factors." Information \& Communication Technologies,IEEE Conference on. IEEE, 2013.

19. Khan, Nawaz, DharaGaurav, and Thomas Kandl. "Performance Evaluation of Levenberg-Marquardt Technique in Error Reduction for Diabetes Condition Classification." Procedia Computer Science, Elsevier 2013.

20. Yin $\mathrm{X}$ and Goudriaan $\mathrm{J}$ "A flexible sigmoid function of determinate growth" department of plant science wageningen university ,2003

21. Pete McCollum, An Introduction to Back-Propagation Neural Networks, Seattle Robotics, n.p, 24 feb 2016, web 1998.

22. Marc Fakhoury, Autistic Spectrum Disorders: A Review Of Clinical Features, Theories And Diagnosis , International journel of development Neuroscience, 2015

23. NurulRidwahMohd Isa, Marina Yusoff , Noor, Elaiza Khalid, Autism Severity Level Detection Using Fuzzy Expert System, IEEE International Symposium on Robotics and Manufacturing Automation,2014

24. Marisela Huerta, Somer L. Bishop, Amie Duncan, Vanessa Hus, Catherine Lord, Application of DSM-5 Criteria for Autism Spectrum Disorder to Three Samples of Children With DSM-IV Diagnoses of Pervasive Developmental Disorders, American Journel of Psychiatry, 2012, 169:1056-1064

25. C.S Kanimozhiselvi_,A. Pratap, PossibilisticLvq Neural Network An Application To Childhood Autism Grading,journel of neural network world, 253-269, 2016.

26. ChathuraPriyankaras How to use Support Vector Machine classifier in OpenCV for Linearly Separable Data sets, JANUARY 2, 2015.

27. Sharma, N., Khullar, V., \&Luhach, A., "Comparative Study of Back-propagation and PSOBased Backpropagation for Anemia Diagnosis in Pregnant Women”, International Journal ofScience, Engineering \& Computer Technology (UGC), Vol. no. 7, Issue no. 1-2, pp. 1-5,2017. 